\title{
Viscodilation of Schlemm's canal for the reduction of IOP via an ab-interno approach
}

This article was published in the following Dove Press journal:

Clinical Ophthalmology

\author{
Mark J Gallardo ${ }^{1,2}$ \\ Richard A Supnet' \\ lqbal Ike K Ahmed ${ }^{3}$ \\ 'El Paso Eye Surgeons, PA, El Paso, \\ TX, USA; ${ }^{2}$ University of Texas Health \\ Sciences Center, San Antonio, TX, \\ USA; ${ }^{3}$ Department of Ophthalmology \\ and Vision Sciences, University of \\ Toronto, Toronto, Ontario, Canada
}

Correspondence: Mark J Gallardo El Paso Eye Surgeons, PA, I20I N Mesa St, Ste G, El Paso, TX 79902, USA

$\mathrm{Tel}+\mathrm{I} 9155420279$

Email gallardomark@hotmail.com
Purpose: The aim of this study was to compare the 1-year efficacy and safety profile of ab-interno canaloplasty $(\mathrm{ABiC})$ when performed as a stand-alone procedure or as an adjunct to cataract extraction in reducing IOP and glaucoma medication dependence.

Patients and methods: This retrospective, comparative, consecutive case series included patients with uncontrolled primary open-angle glaucoma (POAG) who underwent $\mathrm{ABiC}$ as a stand-alone procedure or in conjunction with cataract extraction. Data were collected over a 12-month period. Primary outcome measures were mean lower IOP and mean number of glaucoma medications. Secondary endpoints included surgical and postsurgical complications and secondary interventions.

Results: The study included 75 eyes of 68 patients (mean age: $73.7 \pm 9.9$ years) with a mean baseline IOP of $20.4 \pm 4.7 \mathrm{mmHg}$ on $2.8 \pm 0.9$ medications, which reduced to $13.3 \pm 1.9 \mathrm{mmHg}(\mathrm{n}=73)$ on $1.1 \pm 1.1$ medications at 12 months postoperative (both $P<0.0001$ ). At 12 months, $40 \%$ of eyes were medication free. In the $\mathrm{ABiC} /$ phacoemulsification subgroup ( $\mathrm{n}=34$ eyes), the mean IOP and medication use decreased from $19.4 \pm 3.7 \mathrm{mmHg}$ on $2.6 \pm 1.0$ medications preoperatively to $13.0 \pm 1.8 \mathrm{mmHg}$ on $0.8 \pm 0.2$ medications at 12 months (both $P<0.001$ ). In the stand-alone $\mathrm{ABiC}$ subgroup $(\mathrm{n}=41)$, the mean IOP and medication use decreased from $21.2 \pm 5.3 \mathrm{mmHg}$ on $3.0 \pm 0.7$ medications preoperatively to $13.7 \pm 1.9 \mathrm{mmHg}$ on $1.3 \pm 1.1$ medications at 12 months ( $P=0.001$ and $<0.001$, respectively). No serious adverse events were recorded.

Conclusion: These data demonstrate that $\mathrm{ABiC}$ was effective at reducing IOP and medication use in eyes with uncontrolled POAG with or without cataract surgery.

Keywords: IOP, primary open-angle glaucoma, ab-interno canaloplasty, glaucoma medication

\section{Introduction}

The mainstay of glaucoma treatment, whether eye drops, laser treatment, or surgery, is to lower IOP to preserve optic nerve structure and function of the eye. Traditional glaucoma surgeries such as trabeculectomy and tube shunts work well to lower IOP, but these procedures are not without significant risk. Trabeculectomy, deemed by many surgeons to be the "gold standard" in surgical glaucoma treatments, is associated with numerous immediate and delayed postoperative complications, many of which stem from the creation of a subconjunctival bleb. ${ }^{1-6}$

An alternative bleb-less surgical approach known as canaloplasty has been shown to lower IOP, restoring the eye's conventional outflow pathway., ${ }^{7,8}$ This ab-externo procedure combines concepts of deep sclerectomy and viscocanalostomy in conjunction with a flexible microcatheter that is used to circumnavigate and viscodilate $360^{\circ}$ of Schlemm's canal culminating with a tensioning suture and effectively targets the following three main sites of outflow resistance: pathology of the trabecular 
meshwork (TM), collapse of Schlemm's canal, and collapse or atrophy of the collector channels. ${ }^{9}$

Canaloplasty offers a reduced complication profile compared to trabeculectomy, avoiding long-term, blebrelated complications and simplified postoperative care for both the patient and the physician. ${ }^{10}$ One of the hallmarks of canaloplasty is the placement of the tensioning suture within Schlemm's canal. Abnormalities within the canal that prohibit $360^{\circ}$ of circumnavigation of the microcatheter can preclude placement of the tensioning suture. Interestingly, these patients who were unable to accept the tensioning suture faired equally well as those who did receive the tensioning suture in the degree of IOP and medication reduction postoperatively. ${ }^{11}$ With this finding, we have adopted a modified technique of viscodilating Schlemm's canal via an ab-interno approach, negating the need for large conjunctival and scleral dissections. Ab-interno canaloplasty $(\mathrm{ABiC})$ is an ab-interno minimally invasive glaucoma surgery (MIGS) that achieves $360^{\circ}$ viscodilation of Schlemm's canal through a clear corneal incision without the use of a tensioning suture.

One of the major benefits of $\mathrm{ABiC}$ is the sparing of conjunctiva, leaving it untouched for future procedures if required. It also reduces postoperative recovery as incisions resemble those of a typical cataract extraction, and the addition of $\mathrm{ABiC}$ to phacoemulsification could be considered astigmatically neutral. As with traditional canaloplasty, $\mathrm{ABiC}$ is designed to access, catheterize, and viscodilate all aspects of outflow resistance - the TM, Schlemm's canal, and the distal outflow system beginning with the collector channels.

Prior studies have reported on 1-, 2-, and 3-year results from prospective clinical trials of traditional ab-externo canaloplasty, which showed significant reductions in IOP and glaucoma medication usage in conjunction with an excellent safety profile. ${ }^{12-15}$ Some studies also showed that when coupled with cataract extraction, the lowering effect of canaloplasty is enhanced. However, the role of $\mathrm{ABiC}$ in glaucoma management has not yet been fully delineated. The current study presents 12-month data from a retrospective chart review of patients with primary open-angle glaucoma (POAG) who were treated with $\mathrm{ABiC}$ alone and in conjunction with cataract surgery. The study aims to demonstrate the safety and efficacy of $\mathrm{ABiC}$ in reducing IOP and glaucoma medication dependence in the treatment of POAG.

\section{Patients and methods Study design}

This study presents the 12-month results of a retrospective, single-center comparison of $\mathrm{ABiC}$ as a stand-alone procedure or in conjunction with cataract extraction. Primary endpoints included mean IOP and mean number of antiglaucoma medications at the 12-month follow-up visit. Secondary endpoints included visual acuity, intra- and post-operative complications, and the need for secondary interventions. The study was performed in accordance with the principles stated in the Declaration of Helsinki and was approved by the local Institutional Review Board (Surgical Center of El Paso Medical Executive Committee). Patient consent to review their medical records was not required by the institutional review board as the study was retrospective in nature, but all the data included in the study were non-identifying.

\section{Patient selection}

Patients aged $\geq 18$ years who previously underwent $\mathrm{ABiC}$ alone or in combination with cataract surgery were identified through systematic chart review. Inclusion criteria included a diagnosis of uncontrolled POAG defined as IOP $\geq 18 \mathrm{mmHg}$ and confirmed evidence of characteristic changes to the optic nerve consistent with glaucomatous optic neuropathy and thinning of the retinal nerve fiber layer on ocular coherence tomography (OCT). Patients who had laser trabeculoplasty (LTP) within 1 year of surgery or other angle-based microinvasive glaucoma procedures were excluded. Other exclusion criteria included neovascular disease, uveitis, peripheral anterior synechiae, goniosynechiae, angle recession, and developmental or other forms of secondary glaucoma, such as steroid-induced glaucoma. The protocol allowed previous surgeries that would not interfere with complete circumferential catheterization of Schlemm's canal.

\section{Pre- and postoperative assessment}

All patients had a complete baseline ophthalmic examination prior to surgery that included history of glaucoma, medication use, IOP, best-corrected distance visual acuity (CDVA), gonioscopy, slit-lamp, and fundus examination. Postoperative follow-up examinations were performed at 1 , 3,6 , and 12 months.

\section{Surgical technique}

All procedures were performed under local anesthesia by the same surgeon (MJG) between September 2014 and June 2016. In patients who underwent combined phacoemulsification/ $\mathrm{ABiC}$, cataract surgery was performed first. Following cataract surgery, a side port incision for the iTrack ${ }^{\mathrm{TM}}$ microcatheter was created approximately $90^{\circ}$ away from the nasal drainage angle, typically at the 12 o'clock position. The iTrack ${ }^{\mathrm{TM}}$ microcatheter (Ellex Medical Lasers Ltd., Adelaide, SA, Australia) was then primed with Healon ${ }^{\circledR} /$ Healon $G^{\circledR}$ and 
inserted into the anterior chamber with the catheter tip guided toward the nasal angle. Through the temporal clear corneal incision and under direct visualization using a gonioprism, a small micro-goniotomy in the nasal TM was then created with either a $25 \mathrm{G}$ needle or a straightened Cystotome ${ }^{\circledR}$. Microsurgical forceps were used to grasp the catheter at a slightly oblique angle approximately $1.0-2.0 \mathrm{~mm}$ from its distal end. Schlemm's canal was then intubated with the catheter by gently inserting the catheter through the goniotomy site and circumnavigated $360^{\circ}$; progress and proper catheter location were followed by observing the positional fiber optic red light of the iTrack ${ }^{\mathrm{TM}}$. Following complete circumferential intubation of Schlemm's canal, the microcatheter was slowly withdrawn while steadily injecting two "clicks" of Healon GV every clock hour at a rate of approximately 1 o'clock hour per 1.5 seconds. To prevent the extension of the goniotomy, a Lester hook was placed through the clear corneal incision and was employed to act as a fulcrum; this allowed all catheter tension to be placed on the instrument as opposed to the TM. The dispersive viscoelastic used to maintain the anterior chamber during the procedure was thoroughly evacuated from the anterior chamber before re-pressurizing with balanced salt solution to approximately $20 \mathrm{mmHg}$.

\section{Statistical analysis}

Continuous data were presented as mean \pm SD. Wilcoxon signed-rank test was performed to determine if there was a significant difference in IOP and the number of medications between pre- and postoperative visits. Visual acuity measurements were converted into logarithm of the minimum angle of resolution $(\log$ MAR) notation for the analysis. If visual acuity was recorded as "count fingers", it was transformed as $1.85 \log$ MAR, and if visual acuity was recorded as "Hand Movement", it was transformed as 2.30 logMAR as suggested by Schulze-Bonsel et al. ${ }^{16}$ Statistical analysis was performed using the IBM SPSS Statistics 24 software. The level of statistical significance was set at $P \leq 0.05$.

\section{Results}

\section{Subject demographics/characteristics}

Demographics are summarized in Table 1. The identified cohort included 75 eyes of 68 patients (43 females, 57.3\%) with a mean age of $73.7 \pm 9.9$ years (range 37-91 years). All patients were diagnosed with POAG at different stages: $37.3 \%$ of eyes $(n=28)$ with mild POAG, $16.0 \%$ of eyes $(n=12)$ with moderate POAG, and $38.7 \%$ of eyes $(n=29)$ with severe POAG. Staging of glaucoma severity was defined using the Hodapp-Parrish-Anderson method of classification. ${ }^{17}$
Table I Population demographics ( $\mathrm{n}=75$ eyes)

\begin{tabular}{|l|l|l|}
\hline Age (years) & $\begin{array}{l}\text { Mean } \pm \text { SD } \\
(\text { range })\end{array}$ & $\begin{array}{l}73.7 \pm 9.9 \\
(37-91)\end{array}$ \\
\hline \multirow{3}{*}{ Sex, \% (n) } & Male & $42.6(32)$ \\
\cline { 2 - 3 } & Female & $57.3(43)$ \\
\hline Race, \% (n) & Black & $5.3(4)$ \\
\cline { 2 - 3 } & White & $21.3(16)$ \\
\cline { 2 - 3 } & Hispanic & $73.3(55)$ \\
\hline \multirow{3}{*}{ Type of glaucoma, \% (n) } & POAG & $100(75)$ \\
\cline { 2 - 3 } & $\bullet$ Mild & $37.3(28)$ \\
\cline { 2 - 3 } & - Moderate & $16.0(12)$ \\
\cline { 2 - 3 } & $\bullet$ Severe & $38.7(29)$ \\
\cline { 2 - 3 } & $\bullet$ Unavailable & $8.0(6)$ \\
\hline
\end{tabular}

Abbreviation: POAG, primary open-angle glaucoma.

Out of the 75 eyes, 34 eyes were treated with $\mathrm{ABiC}$ combined with phacoemulsification, and 41 eyes were treated with $\mathrm{ABiC}$ as a stand-alone procedure.

\section{IOP and antiglaucoma medication use}

Figure 1 shows the mean IOP $(\mathrm{mmHg})$ and the mean number of medications for all included eyes. On average, there was a $32.3 \%$ reduction in mean IOP 12 months after surgery $(P<0.001)$. A reduction of greater than $20 \%$ was observed in $84.9 \%$ of eyes. Table 2 shows the distribution of percentage of reduction in IOP at 12 months.

Figure 2 shows the mean number of medications for all included eyes. On average, there was a $60.0 \%$ reduction in medication use at 12 months postoperative $(P<0.001)$. Overall, $40 \%$ of eyes were medication free at this time.

\section{Combined phacoemulsification/ABiC}

The combined subgroup demonstrated a mean IOP reduction of $31.7 \%$ at 12 months postoperative $(P<0.001)$. A reduction of greater than $20 \%$ was observed in $88.2 \%$ of eyes. Table 3 shows the distribution of percentage of reduction in IOP at 12 months for the combined subgroup.

The mean medication use was reduced by $71.1 \%$ at 12 months postoperative $(P<0.001)$. Within this subgroup, $50 \%$ of eyes were medication free.

\section{Stand-alone $\mathrm{ABiC}$}

The stand-alone $\mathrm{ABiC}$ eyes experienced a $32.8 \%$ reduction in IOP at 12 months following surgery. A greater than 20\% reduction was observed in $82.0 \%$ of eyes. Table 4 shows the distribution of percentage reduction in IOP at 12 months. The mean medication use was reduced by $51.1 \%$ at 12 months postoperative $(P<0.001)$. Within this subgroup, $30.8 \%$ of eyes were medication free. 


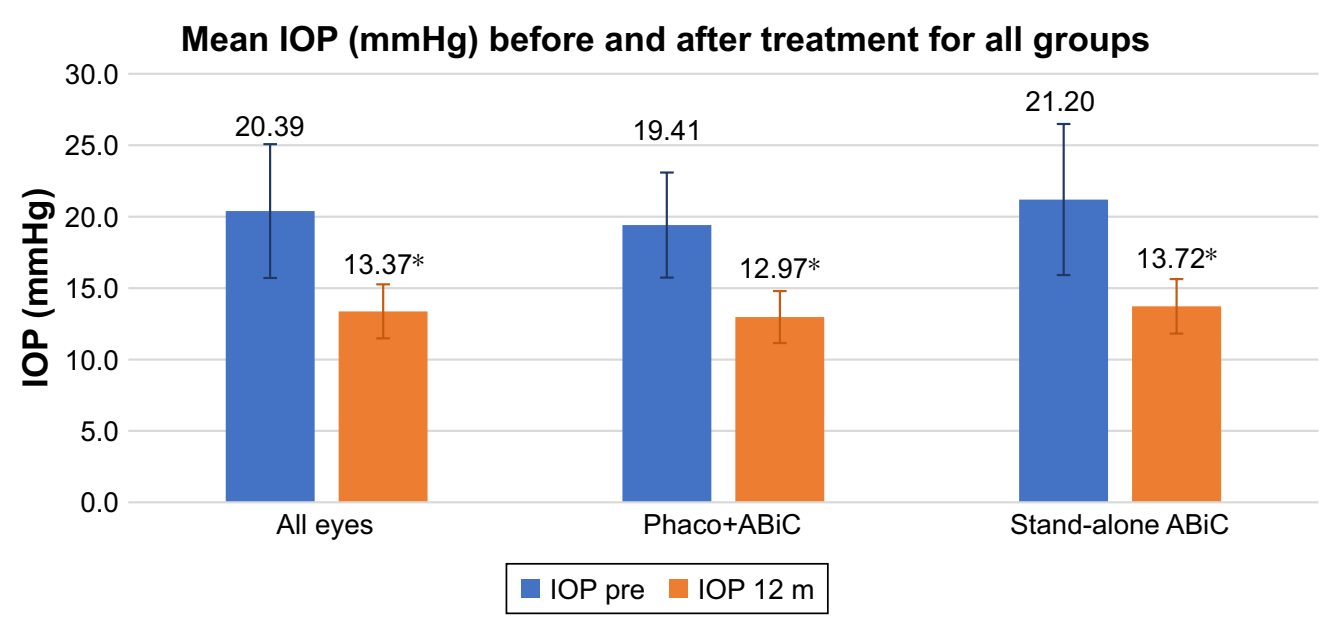

Figure I Mean IOP in mmHg before surgery (blue bars) and I 2 months after surgery (orange bars) for all eyes, for eyes treated with ABiC combined with phacoemulsification, and for eyes treated with stand-alone $\mathrm{ABiC}$.

Note: *Statistically significant difference.

Abbreviations: $\mathrm{ABiC}$, ab-interno canaloplasty; $\mathrm{m}$, months; Phaco, phacoemulsification.

\section{Comparison between the two subgroups}

There were no statistically significant differences between the two subgroups in IOP reduction $(P=0.350)$ or the number of medications required at 1 -year postoperative $(P=0.671)$.

\section{Visual acuity}

Preoperative and 12-month visual acuities are presented in Table 5. Visual acuity was significantly improved at 1-year follow-up for the cohort as a whole, as well as for the combined group $(P \leq 0.001)$. In the stand-alone group, visual acuity was unchanged $(P=0.849)$.

\section{Safety - adverse events}

Overall, the frequency of surgical and postsurgical complications was low with no serious adverse events recorded. Adverse events were limited to intraoperative bleeding at the goniotomy site and postoperative microhyphema development, both of which resolved within 1 week without sequelae. The following two eyes required additional surgery during the 12-month follow-up period: one eye had selective laser trabeculoplasty (SLT) and Express Shunt and one eye had Express Shunt 5 months after surgery. In both cases, the primary surgery failed to lower the IOP. These two eyes were excluded from the postoperative analysis.

\section{Discussion}

In this retrospective, comparative study, $\mathrm{ABiC}$ performed either stand alone or in conjunction with cataract extraction was found to be a safe and effective surgical option for reducing IOP and glaucoma medication dependence in the treatment of POAG. ABiC follows the same dilation principles as traditional canaloplasty, where application of viscoelastic during insertion allows the compressed tissue

Table 2 Distribution of the percentage reduction in IOP for the whole cohort

\begin{tabular}{|l|l|l|l|}
\hline & Frequency & Percentage & $\begin{array}{l}\text { Cumulative } \\
\text { percentage }\end{array}$ \\
\hline No reduction & 0 & 0.00 & 0.00 \\
\hline$<10 \%$ of reduction & 2 & 2.74 & 2.74 \\
\hline$\geq 10$ and $<15 \%$ of reduction & 4 & 5.48 & 8.22 \\
\hline$\geq 15$ and $<20 \%$ of reduction & 5 & 6.85 & 15.07 \\
\hline$\geq 20$ and $<25 \%$ of reduction & 11 & 15.07 & 30.14 \\
\hline$\geq 25$ and $<30 \%$ of reduction & 13 & 17.81 & 47.95 \\
\hline$\geq 30$ and $<35 \%$ of reduction & 6 & 8.22 & 56.16 \\
\hline$\geq 35$ and $<40 \%$ of reduction & 13 & 17.81 & 73.97 \\
\hline$\geq 40 \%$ & 19 & 26.03 & 100.00 \\
\hline
\end{tabular}


Number of medications before and after treatment

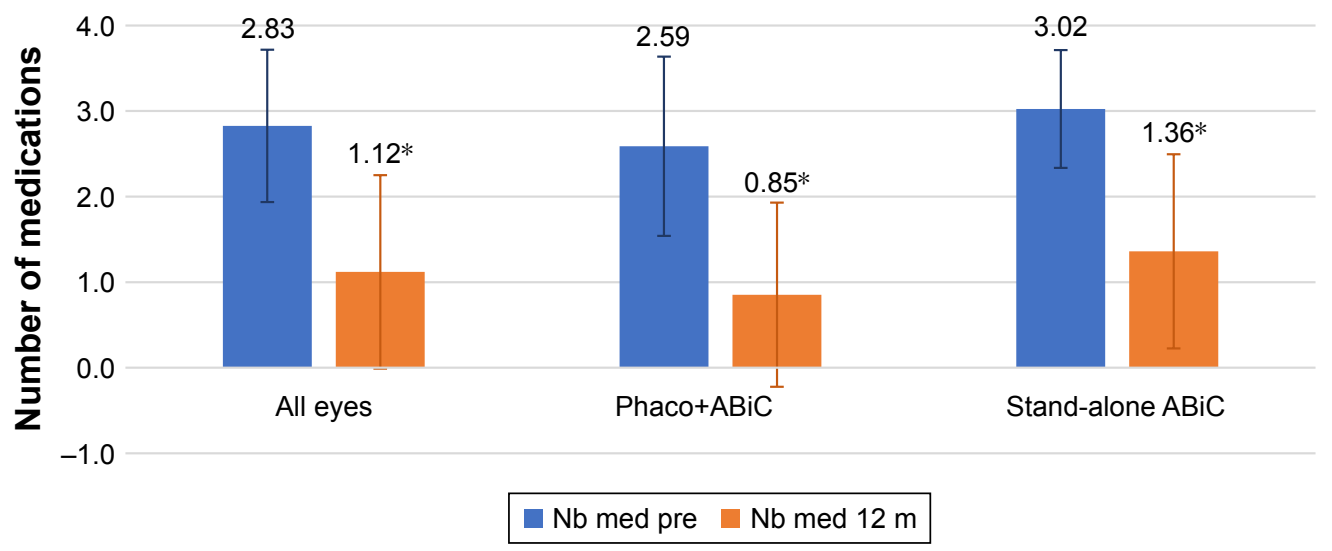

Figure 2 Mean number of medications before surgery (blue bars) and 12 months after surgery (orange bars) for all eyes, for eyes treated with ABiC combined with phacoemulsification, and for eyes treated with stand-alone ABiC.

Note: *Statistically significant difference.

Abbreviations: $\mathrm{ABiC}$, ab-interno canaloplasty; $\mathrm{m}$, months; $\mathrm{Nb}$, number; Phaco, phacoemulsification

planes of the TM and sclera to gently stretch and separate and any herniated TM tissue to withdraw from the collector channels. Although there is debate as to the longevity of this effect, the present study shows promising results at 1 year.

The IOP-lowering ability of ABiC is limited by the episcleral venous-resistant floor and appears to be appropriate for patients with modest IOP targets in the mid-teens. Data from the Early Manifest Glaucoma Trial showed that the risk of disease progression was reduced by $10 \%$ with each millimeter of mercury of IOP reduction from baseline to the first follow-up visit. ${ }^{18}$ Furthermore, in the Advanced Glaucoma Intervention Study, maintaining IOPs consistently below 18 was most protective of progression and maintained a visual field stability. ${ }^{19}$ In the current study, the mean IOP at 12 months post-operatively was $13.5 \pm 1.9 \mathrm{mmHg}$ for the entire cohort, $13.0 \pm 1.8 \mathrm{mmHg}$ for the combined group, and $13.7 \pm 1.9 \mathrm{mmHg}$ for the stand-alone group. All eyes $(100 \%)$ in both groups achieved an IOP lower than the recommended $(18 \mathrm{mmHg}$ ) at 12 months after surgery. In the combined group, postoperative IOP varied between 9 and $16 \mathrm{mmHg}$, and in the stand-alone group, postoperative IOP varied between 9 and $17 \mathrm{mmHg}$. Thus, for patients with modest IOP targets, with or without cataract, $\mathrm{ABiC}$ may be a reasonable and minimally invasive option for reducing medication load unlikely to preclude or affect future potential conjunctival bleb surgeries.

The reductions in IOP achieved in this 12-month study are in line with previously reported ab-externo canaloplasty studies in the literature, and notably in a multicenter prospective trial carried out at 15 clinical sites in USA, Great Britain, and Germany in $2005 .{ }^{20}$ Furthermore, the present results found $\mathrm{ABiC}$ to be effective both combined with cataract surgery and as a stand-alone procedure. It is known that cataract surgery lowers IOP in some glaucoma patients, particularly

Table 3 Distribution of the percentage reduction in IOP for the combined group

\begin{tabular}{|l|l|l|l|}
\hline & Frequency & Percentage & $\begin{array}{l}\text { Cumulative } \\
\text { percentage }\end{array}$ \\
\hline No reduction & 0 & 0.00 & 0.00 \\
\hline$<10 \%$ of reduction & $\mathrm{I}$ & 2.94 & 2.94 \\
\hline$\geq 10$ and $<15 \%$ of reduction & 2 & 5.88 & 8.82 \\
\hline$\geq 15$ and $<20 \%$ of reduction & $\mathrm{I}$ & 2.94 & 11.76 \\
\hline$\geq 20$ and $<25 \%$ of reduction & 6 & 17.65 & 29.41 \\
\hline$\geq 25$ and $<30 \%$ of reduction & 8 & 23.53 & 52.94 \\
\hline$\geq 30$ and $<35 \%$ of reduction & 2 & 5.88 & 58.82 \\
\hline$\geq 35$ and $<40 \%$ of reduction & 7 & 20.59 & 79.41 \\
\hline$\geq 40 \%$ & 7 & 20.59 & 100.00 \\
\hline
\end{tabular}


Table 4 Distribution of the percentage reduction in IOP for the stand-alone group

\begin{tabular}{|l|l|l|l|}
\hline & Frequency & Percentage & $\begin{array}{l}\text { Cumulative } \\
\text { percentage }\end{array}$ \\
\hline No reduction & 0 & 0.00 & 0.00 \\
\hline$<10 \%$ of reduction & 1 & 2.56 & 2.56 \\
\hline$\geq 10$ and $<15 \%$ of reduction & 2 & 5.13 & 7.69 \\
\hline$\geq 15$ and $<20 \%$ of reduction & 4 & 10.26 & 17.95 \\
\hline$\geq 20$ and $<25 \%$ of reduction & 5 & 12.82 & 30.77 \\
\hline$\geq 25$ and $<30 \%$ of reduction & 5 & 12.82 & 43.59 \\
\hline$\geq 30$ and $<35 \%$ of reduction & 4 & 10.26 & 53.85 \\
\hline$\geq 35$ and $<40 \%$ of reduction & 6 & 15.38 & 69.23 \\
\hline$\geq 40 \%$ & 12 & 30.77 & 100.00 \\
\hline
\end{tabular}

those with a higher preoperative IOP. ${ }^{21}$ The physiological reasons behind this phenomenon are not entirely clear, but cataract surgery, such as $\mathrm{ABiC}$, increases aqueous outflow, perhaps by improving the function of the TM, Schlemm's canal and collector channels. Regardless, the efficacy and safety of $\mathrm{ABiC}$ were the same when performed combined with phacoemulsification or as a stand-alone procedure, lending evidence to its utility. These findings suggest that $\mathrm{ABiC}$ is a comprehensive MIGS capable of restoring the outflow pathways in isolation when applicable.

The study is limited by the characteristic confines of retrospective research, including selection bias and variable follow-up, although all eyes in this study had 12-month follow-up data. Furthermore, the combination cataract surgery results are confounded by the IOP-lowering effect of cataract surgery. The precise mechanism of the ability of $\mathrm{ABiC}$ to reduce IOP requires further exploration. With a small sample size, it was challenging to report low frequency complications. Nevertheless, the minimally invasive ab-interno physiological approach and these early results likely point to a very safe procedure. Our study results are quite short term and with a small group of patients but exhibit promising results for this new procedure that highlights the need for longer term, prospective multicenter trials with a larger sample size.

Table 5 Mean visual acuity preoperatively and 12 months postoperatively

\begin{tabular}{|l|l|l|l|}
\hline \multirow{2}{*}{} & \multicolumn{2}{|l|}{ Visual acuity (logMAR) } & \multirow{2}{*}{ P-value } \\
\cline { 2 - 3 } & Preoperative & Postoperative & \\
\hline All eyes & $0.50 \pm 0.47$ & $0.35 \pm 0.49$ & 0.002 \\
\hline Combined subgroup & $0.58 \pm 0.41$ & $0.29 \pm 0.41$ & 0.001 \\
\hline Stand-alone subgroup & $0.44 \pm 0.52$ & $0.41 \pm 0.56$ & 0.849 \\
\hline
\end{tabular}

Abbreviation: logMAR, logarithm of the minimum angle of resolution.

\section{Conclusion}

A further evolution of canaloplasty, $\mathrm{ABiC}$ endeavors to be a restorative MIGS procedure that enhances the function of the eye's natural outflow system without the need for a scleral incision or tensioning suture. The ABiC approach spares conjunctival manipulation for future procedures if needed. Furthermore, given the minimally invasive, nondestructive nature of the procedure, further angle surgery can be performed if necessary including LTP, goniotomy, trabeculotomy, or trabecular micro-bypass. And as other minimally invasive glaucoma surgeries, $\mathrm{ABiC}$ has a limited postoperative recovery compared to other more invasive filtering procedures, lending itself to being adjunctively added to cataract extraction.

\section{Disclosure}

MJG, MD, is a consultant/clinical investigator for Ellex. IIKA is a consultant for Ellex. The authors report no other conflicts of interest in this work.

\section{References}

1. Jones E, Clarke J, Khaw PT. Recent advances in trabeculectomy technique. Curr Opin Ophthalmol. 2005;16(2):107-113.

2. Borisuth NS, Phillips B, Krupin T. The risk profile of glaucoma filtration surgery. Curr Opin Ophthalmol. 1999;10(2):112-116.

3. Gedde SJ, Herndon LW, Brandt JD, Budenz DL, Feuer WJ, Schiffman JC. Surgical complications in the Tube Versus Trabeculectomy Study during the first year of follow-up; the TubeVersus Trabeculectomy Study Group. Am J Ophthalmol. 2007;143:23-31.

4. Scott IU, Greenfield DS, Schiffman J, et al. Outcomes of primary trabeculectomy with the use of adjunctive mitomycin. Arch Ophthalmol. 1998;116(3):286-291.

5. Jampel HD, Musch DC, Gillespie BW, Lichter PR, Wright MW, Guire KE. Perioperative complications of trabeculectomy in the Collaborative Initial Glaucoma Treatment Study (CIGTS); the Collaborative Initial Glaucoma Treatment Study Group. Am J Ophthalmol. 2005; $140: 16-22$.

6. Edmunds B, Thompson JR, Salmon JF, Wormald RP. The National Survey of Trabeculectomy. III. Early and late complications. Eye (Lond). 2002; 16(3):297-303. 
7. Bull H, von Wolff K, Körber N, Tetz M. Three-year canaloplasty outcomes for the treatment of open-angle glaucoma: European study results. Graefes Arch Clin Exp Ophthalmol. 2011;249(10):1537-1545.

8. Grieshaber MC, Pienaar A, Olivier J, Stegmann R. Canaloplasty for primary open-angle glaucoma: long-term outcome. Br J Ophthalmol. 2010;94(11):1478-1482.

9. Stegmann R, Pienaar A, Grieshaber MC. Schlemm's canal surgery: restoring physiological aqueous outflow. Glaucoma Therapy - State of the Art. In: Grieshaber MC, Orgul S, Flammer J, editors. Basel: Association for Continuing Education in Ophthalmology; 2009.

10. Zahid S, Musch DC, Niziol LM, Lichter PR. Collaborative Initial Glaucoma Treatment Study Group. Risk of endophthalmitis and other long-term complications of trabeculectomy in the Collaborative Initial Glaucoma Treatment Study (CIGTS). Am J Ophthalmol. 2013; 155(4):674-680.

11. Lewis RA, von Wolff K, Tetz M, et al. Canaloplasty: Three-year results of circumferential viscodilation and tensioning of Schlemm canal using a microcatheter to treat open-angle glaucoma. J Cataract Refract Surg. 2011;37(4):682-690.

12. Lewis RA, von Wolff $\mathrm{K}$, Tetz M, et al. Canaloplasty: circumferential viscodilation and tensioning of Schlemm's canal using a flexible microcatheter for the treatment of open-angle glaucoma in adults: interim clinical study analysis. J Cataract Refract Surg. 2007;33(7): 1217-1226.

13. Lewis RA, von Wolff K, Tetz M, et al. Canaloplasty: circumferential viscodilation and tensioning of Schlemm canal using a flexible microcatheter for the treatment of open-angle glaucoma in adults: two-year interim clinical study results. J Cataract Refract Surg. 2009;35(5): 814-824.
14. Grieshaber MC, Fraenkl S, Schoetzau A, Flammer J, Orgül S. Circumferential viscocanalostomy and suture canal distension (canaloplasty) for whites with open-angle glaucoma. J Glaucoma. 2011;20(5):298-302.

15. Lewis RA, von Wolff K, Tetz M, et al. Canaloplasty: Three-year results of circumferential viscodilation and tensioning of Schlemm canal using a microcatheter to treat open-angle glaucoma. J Cataract Refract Surg. 2011;37(4):682-690.

16. Schulze-Bonsel K, Feltgen N, Burau H, Hansen L, Bach M. Visual acuities "hand motion" and "counting fingers" can be quantified with the freiburg visual acuity test. Invest Ophthalmol Vis Sci. 2006;47(3): 1236-1240.

17. Hodapp E, Parrish RK II, Anderson DR. Clinical Decisions in Glaucoma. St Louis: The CV Mosby Co; 1993:52-61.

18. Leske MC, Heijl A, Hussein M, Bengtsson B, Hyman L, Komaroff E. Early Manifest Glaucoma Trial Group. Factors for glaucoma progression and the effect of treatment: the early manifest glaucoma trial. Arch Ophthalmol. 2003;121(1):48-56.

19. The Advanced Glaucoma Intervention Study (AGIS): 7. The relationship between control of intraocular pressure and visual field deterioration. The AGIS Investigators. Am J Ophthalmol. 2000;130(4):429-440.

20. Edmunds B, Thompson JR, Salmon JF, Wormald RP. The National Survey of Trabeculectomy. II. Variations in operative technique and outcome. Eye (Lond). 2001;15(Pt 4):441-448.

21. Berdahl JP. Cataract surgery to lower intraocular pressure. Middle East Afr J Ophthalmol. 2009;16(3):119-122.
Clinical Ophthalmology

\section{Publish your work in this journal}

Clinical Ophthalmology is an international, peer-reviewed journa covering all subspecialties within ophthalmology. Key topics include: Optometry; Visual science; Pharmacology and drug therapy in eye diseases; Basic Sciences; Primary and Secondary eye care; Patient Safety and Quality of Care Improvements. This journal is indexed on

Submit your manuscript here: http://www.dovepress.com/clinical-ophthalmology-journal

\section{Dovepress}

PubMed Central and CAS, and is the official journal of The Society of Clinical Ophthalmology (SCO). The manuscript management system is completely online and includes a very quick and fair peer-review system, which is all easy to use. Visit http://www.dovepress.com/ testimonials.php to read real quotes from published authors. 